\title{
Role of Prostatic Stem Cell Antigen (PSCA) and Snail in Different Prostatic Lesions (An immunohistochemical Study)
}

Marwa S. Abd Allah, Nancy Abo Elgheit Dawood, Ranih Z. Amer, Taghreed Abd Elsamea, Abd Ellatif M. Elbalshy

\begin{abstract}
Department of pathology, Benha faculty of medicine, Benha University, Egypt.

Correspondence to: Nancy Abo Elgheit Dawood, Department of pathology, Benha faculty of medicine, Benha University, Egypt.
\end{abstract}

Email: nancyaboelgheit@gmail.com

Received: 12 December 2020 Accepted: 31 January 2021

\begin{abstract}
:
Background: Prostatic carcinoma $(\mathrm{PCa})$ represents the second most common cancer, and the fifth leading cause of cancer death among males worldwide. PSCA is a GPI-anchored cell surface protein. It belongs to the Thy-1/Ly-6 family which shows a functional diversity ranging from $\mathrm{T}$-cell activation to apoptosis regulation. Snail is one of zinc finger proteins which are transcriptional repressors of E-cadherin. Aim: To study PSCA and Snail expression in different prostatic lesions to evaluate their roles in PCa. Material and Methods: This retrospective study was done upon 80 different prostatic lesions; 17 cases of BPH, 13 cases of HGPIN, and 50 cases of PCa. PSCA and Snail immunostaining was done and assessed for each case. Results: There was a highly significant statistical correlation between both PSCA and Snail expressions and histopathological type (Pvalue<0.01). PSCA expression showed a highly significant statistical correlation with Gleason score, tumor grade and stage (P-value<0.01), and a significant correlation with PSA, and peri-
\end{abstract} neural invasion $(\mathrm{P}$-value $<0.05)$. Snail expression showed a highly significant statistical correlation with Gleason score and tumor grade (P-value $<0.01)$, and a significant correlation with lymph node metastasis and tumor stage (P-value<0.05). There was a highly significant statistical correlation between PSCA and Snail immune-expression (P-value<0.01). Conclusion: PSCA and Snail expressions correlate with the most important prognostic clinicopathological variables in PCa, thus they may represent a useful predictor of prognosis.

Keyword: Prostatic carcinoma, PSCA, Snail. 

Abbreviations: (PCa): Prostatic carcinoma, (PSCA): Prostatic stem cell antigen, (GPI): Glycosylphosphatidylinositol, (BPH): Benign prostatic hyperplasia, (HGPIN): High grade prostatic intraepithelial neoplasia.

\section{Introduction}

Benign prostatic hyperplasia is one of the most common prostatic diseases that increased in incidence with advanced age (1).

Prostatic intraepithelial neoplasia (PIN) is a neoplastic proliferation of prostatic epithelial cells confined to preexisting prostatic acini (2). Many morphologic and molecular data support that HGPIN is a precursor to PCa as HGPIN is usually seen in association with carcinoma, as well as dominates in the peripheral zone (3).

Prostatic carcinoma ( $\mathrm{PCa}$ ) is the second most frequent malignancy and the fifth leading cause of cancer death in men worldwide (4). It has a significant geographic variation with the highest incidence in North America (5), while lower incidence is reported in Asian and Arabic populations (6). In Egypt, according to National Cancer Institute registry, $\mathrm{PCa}$ represents most of male genital cancers $(60.7 \%)$ in the last 10 years with median age 72.8 years $(\mathbf{7})$.
Prostatic carcinoma has many risk factors as advancing age. The risk begins at 50 years old, reaching its peak in the 7th-8th decades. Also, inherited gene mutations such as BRCA2 or HOXB13, raise the risk (8).

Diagnosis and treatment of $\mathrm{PCa}$ become challenging (9). Clinicopathological factors like Gleason grade, PSA level, clinical and pathological stage were used to assess the prognosis, but instability and susceptibility of these factors still exist. Therefore, new biomarkers are needed (10).

Prostate stem cell antigen (PSCA) is a small, glycosylphosphatidylinositol anchored cell surface protein belonging to the Thy-1/Ly- 6 family. Although it was designated as a 'stem cell antigen' localized to the basal cell epithelium, and stem cell compartment of prostatic epithelium, PSCA now is expressed in differentiating rather than stem cells. PSCA may be a new marker associated with transformation of prostatic cells and tumorigenesis (11).

Epithelial-mesenchymal transition (EMT) is suggested to promote PCa metastasis. EMT is a complex process in which cells lose their epithelial characteristics and acquire mesenchymal features (12). 
It is regulated by numerous pathways and signaling molecules that converge to downregulate the expression of junction molecule E-cadherin. The major transcriptional repressors of E-cadherin are zinc finger family proteins as Snail (SNAIL1 in drosophila) and Slug (13). Snail; as a transcription factor can down-regulate Ecadherin (cell-cell adhesion molecule), and repress tight junction proteins like claudin (14).

PCa cell lines were studied PCa cell lines, and it was found that PSCA knockdown led to decrease the metastatic potentials of $\mathrm{PCa}$ cells, down-regulate E-cadherin, and upregulate the mesenchymal marker vimentin, and although the EMT-related genes like Slug and Twist were elevated, Snail was down-regulated. So, PSCA knockdown led to Snail down-regulation. This suggests that PSCA may have a role in regulating the function and expression of Snail; however the mechanism remains to be investigated.

This study aimed to evaluate the immunohistochemical expression of PSCA and Snail in different prostatic lesions and correlate the results with clinico-pathological data to clarify their diagnostic and prognostic role in prostatic carcinoma.

\section{Material and Methods}

This retrospective study is performed on formalin fixed, paraffin embedded biopsy specimens, from 80 different prostatic lesions, including 17 cases of $\mathrm{BPH}, 13$ cases of HGPIN, and 50 cases of PCa collected from Pathology Department, and Early Cancer Detection Unit (ECDU), Faculty of Medicine, Benha University, between the years 2014 and 2019. The specimens included 25 cases of radical prostatectomy, 31 cases of prostatic chips, and 24 cases of prostatic cores. The study was approved by the Research Ethical Committee of Faculty of Medicine, Benha University.

\section{A- Histopathological Examination:}

Hematoxylin and eosin-stained slides of all cases were revised by two pathologists to confirm the diagnosis, and evaluate different histopathological data of PCa such as grade and capsular, peri-neural, and lymphovascular invasions. The histopathological type was reviewed according to the 2016 WHO classification (16). Each case of PCa was graded according to the Gleason scoring system based on the guidelines of the 2019 International Society of Urological Pathology (ISUP) consensus conference on Gleason grading of PCa (grade group 
I=score 6 , grade group II (score $3+4)$, grade group III (score $4+3$ ), grade group IV (score 8) and grade group V (score 9-10) (17). Tumor stage was defined according to the TNM system applied by the American Joint Committee on Cancer (AJCC), 2017 (18).

\section{B-Immunohistochemical Procedure:}

From formalin-fixed, paraffin-embedded tissue blocks, 3-4 micron tissue sections were obtained on coated slides. After xylene de-paraffinization, the sections were rehydrated in descending grades of alcohol then in distilled water. Antigen retrieval was done by using $10 \mathrm{mmol} / \mathrm{L}$ citrate monohydrate buffer ( $\mathrm{pH}$ 6.0) and heated for 15 minutes in microwave.

The endogenous peroxidase activity was inactivated by incubation in $3 \%$ hydrogen peroxide $\left(\mathrm{H}_{2} \mathrm{O}_{2}\right)$ for 15 minutes then washing by distilled water. Slides then were incubated with the primary polyclonal antibodies, PSCA and Snail at a dilution of $1: 100 \quad(0.1 \mathrm{mg} / \mathrm{ml} \quad$ concentration, Chongqing, YPA1898, China and 0.1mg/ml concentration, Chongqing, YPA1657, China respectively) overnight. Immunodetection was executed using a standard labeled streptavidin-biotin system (Dako Cytomation, Denmark, A/S).
Immunoreaction was seen by adding DAB as a chromagen. Counterstaining of slides was done with Mayer hematoxylin for 1-2 minutes and dehydrated in ascending alcohol. The slides were cleared in xylene for three changes and covered.

\section{Negative \& positive controls:}

According to manufacture instructions, breast adenocarcinoma sections, were used as a positive control for PSCA (19), and colon carcinoma sections, were used as a positive control for Snail (20).

For negative controls, samples were treated as described above, but the primary antibody was replaced by BSA solution in phosphatebuffered saline (PBS) (19\&20).

\section{Immunostaining evaluation:}

PSCA expression was detected as cytoplasmic brown coloration. According to Ruan et al. (20), the staining extent (percentage of positive cells) was quantified as (Score 0: no staining, (Score 1+) weak expression: ( $<25 \%$ positive cells), (score $2+$ ) moderate expression: (25-50\% positive cells), and (score 3+) strong expression: (>50\% positive cells).

Positive immunostaining for Snail is nuclear brown coloration. The expression was 
evaluated by an immunoreactivity score depending on the extent.

It was graded from 0-3 based on percentage of positive cells as: score 0 as negative (<10\% positive cells), Score $1 \quad(10-30 \%$ positive cells) as weakly positive, Score 2 (30-70\% positive cells) as moderately positive, and Score 3 (>70\% positive cells) as strongly positive (19).

Statistical analysis: Results were analyzed by SPSS (version 20) statistical package for Microsoft windows. The Pearson correlation coefficient was used for statistical analysis. $\mathrm{P}$ value $<0.05$ was considered statistically significant, and $\mathrm{P}$ value $<0.01$ as highly statistically significant.

Receiver-operating characteristic (ROC) curve was used to predict sensitivity, specificity and accuracy of immunohistochemical score in differentiating between cancerous and noncancerous prostatic lesions.

\section{Results}

\section{1-Clinical results:}

This study was carried upon 80 cases of different prostatic lesions, 17 cases $(21.25 \%)$ were of $\mathrm{BPH}, 13$ cases $(16.25 \%)$ were of HGPIN, and 50 cases $(62.5 \%)$ were of PCa. The age of studied cases ranged between 38-91 years old, with the mean age of BPH, HGPIN, and PCa cases was 60, 65, and 65.5 years respectively. Also, the mean PSA level in BPH, HGPIN, and PCa cases was $(7.3,13.1$, and $23.5 \mathrm{ng} / \mathrm{ml}$ respectively).

\section{2-Histopathological results:}

The PCa cases included 12 cases of grade group I, 14 cases of grade group II, 8 cases of grade group III, 7 cases of grade group IV, and 9 cases of grade group V. Regards the stage; there were 9 cases of stage I, 20 cases of stage II, 11 cases of stage III, and 10 cases of stage IV.

Gleason grade groups of $\mathrm{PCa}$ showed a highly significant statistical correlation with pathologic T (pT), and tumor stage (Pvalue<0.01), and a significant statistical correlation with patient's age, PSA, perineural, and lymphovascular invasion (Pvalue<0.05). But, showed insignificant statistical correlation with capsular invasion (in prostatectomy specimens), lymph node, and distant metastasis Table (1).

\section{3-Immunohistochemical results:}

\section{* PSCA expression in studied cases:}

Out of the 80 cases studied, 27 cases (33.75\%) showed weak (1+) expression, 25 cases $(31.25 \%)$ showed moderate (2+) expression, 17 cases $(21.25 \%)$ showed strong (3+) expression and 11 cases $(13.75 \%)$ were 
negative. PSCA expression showed a highly significant statistical correlation with histopathological type of the lesion (Pvalue<0.01) (Figure 1), a significant statistical correlation with PSA (Pvalue $<0.05$ ), and insignificant correlation with patient's age (P-value>0.05).

Relation between the score of PSCA expression and clinico-pathological parameters of prostatic carcinoma:

PSCA expression in $\mathrm{PCa}$ cases showed a highly significant statistical correlation with Gleason score, tumor grade, stage and pathologic $\mathrm{T}$ (P-value<0.01), a significant statistical correlation with lymph node metastasis, peri-neural and lymphovascular invasions (P-value $<0.05$ ), and insignificant statistical correlation with capsular invasion (in prostatectomy specimens), and distant metastasis (P-value>0.05) (Table 2 and Figure 2).

\section{$\underline{\text { Snail expression in studied cases: }}$}

Out of the 80 cases, 25 cases $(31.25 \%)$ showed weak (score 1) expression, 19 cases (23.75\%) showed moderate (score 2) expression, 17 cases $(21.25 \%)$ showed strong (score 3) expression, and 19 cases $(23.75 \%)$ were negative. Snail expression showed a highly significant statistical correlation with histopathological type of the lesion (P- value $<0.01$ ) (Figure 3), and insignificant correlation with PSA and patient's age (Pvalue $>0.05)$.

Relation between the score of Snail expression and clinico-pathological parameters of prostatic carcinoma:

Snail expression showed a highly significant statistical correlation with Gleason score, and tumor grade (P-value<0.01), a significant statistical correlation with pathologic $\mathrm{T}$, lymph node metastasis, and stage (Pvalue $<0.05$ ), and insignificant statistical correlation with distant metastasis, capsular (in prostatectomy specimens), peri-neural and lymphovascular invasion (P-value $>0.05)$ (Table 3 and Figure 4).

\section{ROC curve results:}

Receiver-operating characteristic (ROC) curve was used to predict sensitivity, specificity and accuracy of PSCA and Snail immunohistochemical score in differentiating between cancerous and noncancerous prostatic lesions.

Regards PSCA, sensitivity was 58\%, specificity was $63.3 \%$, and PPV was 72.5 . However, Snail showed 62\% sensitivity, 83.3\% specificity, and PPV was 86.1 , so Snail is more valid than PSCA in differentiating between cancerous and noncancerous prostatic lesions (Figures 5, 6 and Table 4). 
Relation between the score of PSCA and Snail expression in the studied cases:

There was a highly significant statistical correlation between the score of PSCA and
Snail expression in the studied different prostatic lesions (P-value $<\mathbf{0 . 0 1})$ (Table 5).

Table (1): Relation between Gleason grade groups of PCa and other clinic-pathological parameters:

\begin{tabular}{|c|c|c|c|c|c|c|c|c|}
\hline \multirow[t]{2}{*}{ Parameters } & \multirow{2}{*}{$\begin{array}{c}\text { Categories } \\
\text { of the } \\
\text { parameter }\end{array}$} & \multirow{2}{*}{$\begin{array}{c}\text { No. } \\
\text { of } \\
\text { cases }\end{array}$} & \multicolumn{5}{|c|}{ Gleason grade groups of PCa } & \multirow[b]{2}{*}{ P-value } \\
\hline & & & Grade I & $\begin{array}{l}\text { Grade } \\
\text { II }\end{array}$ & $\begin{array}{c}\text { Grade } \\
\text { III }\end{array}$ & $\begin{array}{c}\text { Grade } \\
\text { IV }\end{array}$ & $\begin{array}{l}\text { Grade } \\
\text { V }\end{array}$ & \\
\hline \multirow{3}{*}{ Age } & $<40$ & 6 & $\begin{array}{c}2 \\
(33.3 \%)\end{array}$ & $2(33.3 \%)$ & 1 (16.7\%) & $\begin{array}{c}1 \\
(16.7 \%)\end{array}$ & 0 & \multirow{3}{*}{$<0.05^{*}$} \\
\hline & $40-65$ & 23 & $\begin{array}{c}6 \\
(26.1 \%)\end{array}$ & 9 (39.1\%) & $4(17.4 \%)$ & $2(8.7 \%)$ & $2(8.7 \%)$ & \\
\hline & $>65$ & 21 & $4(19 \%)$ & $3(14.3 \%)$ & $3(14.3 \%)$ & $4(19 \%)$ & $\begin{array}{c}7 \\
(33.4 \%)\end{array}$ & \\
\hline \multirow{2}{*}{ Serum PSA level } & $\begin{array}{c}4-10 \\
\mathrm{ng} / \mathrm{ml}\end{array}$ & 24 & $\begin{array}{c}9 \\
(37.5 \%)\end{array}$ & $6(25 \%)$ & $5(20.8 \%)$ & $\begin{array}{c}3 \\
(12.5 \%)\end{array}$ & $1(4.2 \%)$ & \multirow{2}{*}{$<0.05^{*}$} \\
\hline & $>10 \mathrm{ng} / \mathrm{ml}$ & 26 & $\begin{array}{c}3 \\
(11.5 \%)\end{array}$ & $8(30.8 \%)$ & $3(11.5 \%)$ & $\begin{array}{c}4 \\
(15.4 \%)\end{array}$ & $\begin{array}{c}8 \\
(30.8 \%)\end{array}$ & \\
\hline \multirow{2}{*}{$\begin{array}{l}\text { Capsular invasion } \\
\text { in prostatectomy } \\
\text { specimens only }\end{array}$} & Present & $17 / 25$ & $\begin{array}{c}3 \\
(17.6 \%)\end{array}$ & $\begin{array}{c}3 \\
(17.6 \%)\end{array}$ & $4(23.6 \%)$ & $\begin{array}{c}3 \\
(17.6 \%)\end{array}$ & $\begin{array}{c}4 \\
(23.6 \%)\end{array}$ & \multirow[t]{2}{*}{$>0.05$} \\
\hline & Absent & $8 / 25$ & 0 & $2(25 \%)$ & $2(25 \%)$ & $2(25 \%)$ & $2(25 \%)$ & \\
\hline \multirow{2}{*}{$\begin{array}{l}\text { Peri-neural } \\
\text { invasion }\end{array}$} & Present & 13 & 0 & $\begin{array}{c}4 \\
(30.8 \%)\end{array}$ & $2(15.3 \%)$ & $\begin{array}{c}3 \\
(23.1 \%)\end{array}$ & $\begin{array}{c}4 \\
(30.8 \%)\end{array}$ & \multirow{2}{*}{$<0.05^{*}$} \\
\hline & Absent & 37 & $\begin{array}{c}12 \\
(32.5 \%)\end{array}$ & $\begin{array}{c}10 \\
(27 \%)\end{array}$ & $6(16.2 \%)$ & $\begin{array}{c}4 \\
(10.8 \%)\end{array}$ & $\begin{array}{c}5 \\
(13.5 \%)\end{array}$ & \\
\hline \multirow{2}{*}{$\begin{array}{c}\text { Lymphovascular } \\
\text { invasion }\end{array}$} & Present & 20 & $3(15 \%)$ & $4(20 \%)$ & $3(15 \%)$ & $4(20 \%)$ & $6(30 \%)$ & \multirow[b]{2}{*}{$<0.05 *$} \\
\hline & Absent & 30 & $9(30 \%)$ & $\begin{array}{c}10 \\
(33.3 \%)\end{array}$ & $5(16.7 \%)$ & $3(10 \%)$ & $3(10 \%)$ & \\
\hline \multirow{2}{*}{ Pathologic $T(p T)$} & $p T_{2}$ & 29 & $\begin{array}{c}12 \\
(41.4 \%)\end{array}$ & $\begin{array}{c}10 \\
(34.5 \%)\end{array}$ & $4(13.8 \%)$ & $2(6.9 \%)$ & $1(3.4 \%)$ & \multirow{2}{*}{$<0.01 * *$} \\
\hline & $p T_{3}$ & 21 & 0 & $4(19 \%)$ & $4(19 \%)$ & $\begin{array}{c}5 \\
(23.9 \%)\end{array}$ & $\begin{array}{c}8 \\
(38.1 \%)\end{array}$ & \\
\hline \multirow{2}{*}{$\begin{array}{l}\text { Lymph Node } \\
\text { metastasis (N) }\end{array}$} & Present & 7 & 0 & $1(14.2 \%)$ & $2(28.6 \%)$ & $\begin{array}{c}2 \\
(28.6 \%)\end{array}$ & $\begin{array}{c}2 \\
(28.6 \%)\end{array}$ & \multirow{2}{*}{$>0.05$} \\
\hline & Absent & 43 & $\begin{array}{c}12 \\
(27.9 \%)\end{array}$ & $\begin{array}{c}13 \\
(30.2 \%)\end{array}$ & $6(14 \%)$ & $\begin{array}{c}5 \\
(11.6 \%)\end{array}$ & $\begin{array}{c}7 \\
7 \\
(16.3 \%)\end{array}$ & \\
\hline \multirow[b]{2}{*}{$\begin{array}{l}\text { Distant metastasis } \\
\text { (M) }\end{array}$} & Present & 4 & 0 & $1(25 \%)$ & 0 & $1(25 \%)$ & $2(50 \%)$ & \multirow[b]{2}{*}{$>0.05$} \\
\hline & Absent & 46 & $\begin{array}{c}12 \\
(26.1 \%)\end{array}$ & $\begin{array}{c}13 \\
(28.3 \%)\end{array}$ & $8(17.4 \%)$ & $6(13 \%)$ & $\begin{array}{c}7 \\
(15.2 \%)\end{array}$ & \\
\hline \multirow{4}{*}{ Stage of PCa } & I & 9 & $\begin{array}{c}5 \\
(55.6 \%)\end{array}$ & 4 (44.4\%) & 0 & 0 & 0 & \multirow{5}{*}{$<0.01 * *$} \\
\hline & II & 20 & $7(35 \%)$ & $6(30 \%)$ & $4(20 \%)$ & $2(10 \%)$ & $1(5 \%)$ & \\
\hline & III & 11 & 0 & $2(18.2 \%)$ & $3(27.3 \%)$ & $\begin{array}{c}2 \\
(18.2 \%)\end{array}$ & $\begin{array}{c}5 \\
(45.5 \%)\end{array}$ & \\
\hline & IV & 10 & 0 & $2(20 \%)$ & $1(10 \%)$ & $3(30 \%)$ & $3(30 \%)$ & \\
\hline \multicolumn{2}{|c|}{ Total number of PCa cases } & 50 & $\begin{array}{l}12 \\
(24 \%)\end{array}$ & $\begin{array}{l}14 \\
(28 \%)\end{array}$ & $8(16 \%)$ & $7(14 \%)$ & $9(18 \%)$ & \\
\hline
\end{tabular}


Table (2): Relation between the score of PSCA expression and other clinic-pathological parameters:

\begin{tabular}{|c|c|c|c|c|c|c|c|}
\hline \multirow{2}{*}{$\begin{array}{c}\text { Clinico-pathological } \\
\text { parameter }\end{array}$} & \multirow{2}{*}{$\begin{array}{c}\text { Categories } \\
\text { of the } \\
\text { parameter }\end{array}$} & \multirow[b]{2}{*}{$\begin{array}{l}\text { No. of } \\
\text { cases }\end{array}$} & \multicolumn{3}{|c|}{ Score of PSCA expression } & \multirow[b]{2}{*}{$\begin{array}{c}\text { Strong } \\
(3+)\end{array}$} & \multirow[b]{2}{*}{ P-value } \\
\hline & & & Negative & Weak (1+) & $\begin{array}{c}\text { Moderate } \\
(2+)\end{array}$ & & \\
\hline \multicolumn{2}{|l|}{ Studied cases } & 80 & $\begin{array}{c}11 / 80 \\
(13.75 \%)\end{array}$ & $\begin{array}{c}27 / 80 \\
(33.75 \%)\end{array}$ & $\begin{array}{c}25 / 80 \\
(31.25 \%)\end{array}$ & $\begin{array}{c}17 / 80 \\
(21.25 \%)\end{array}$ & \multirow{5}{*}{$<0.01$} \\
\hline \multirow{4}{*}{$\begin{array}{l}\text { Histopathological type } \\
\text { of the prostatic lesion }\end{array}$} & BPH & 17 & $4(23.5 \%)$ & $10(58.8 \%)$ & $3(17.6 \%)$ & 0 & \\
\hline & HGPIN & 13 & $1(7.7 \%)$ & $4(30.7 \%)$ & $6(46.2 \%)$ & $2(15.4 \%)$ & \\
\hline & $P C a$ & 50 & $6(12 \%)$ & $13(26 \%)$ & $16(32 \%)$ & $15(30 \%)$ & \\
\hline & $<40$ years & 8 & 3 (37.5\%) & $1(12.5 \%)$ & 3 (37.5\%) & $1(12.5 \%)$ & \\
\hline \multirow{3}{*}{ Age } & $40-65$ years & 34 & $2(5.9 \%)$ & $17(50 \%)$ & $10(58.8 \%)$ & $5(14.7 \%)$ & \multirow{2}{*}{$>0.05$} \\
\hline & $>65$ years & 38 & $6(15.8 \%)$ & $9(23.7 \%)$ & $12(31.6 \%)$ & $\begin{array}{c}11 \\
(28.9 \%)\end{array}$ & \\
\hline & $<4 \mathrm{ng} / \mathrm{ml}$ & 5 & $1(20 \%)$ & $2(40 \%)$ & $2(40 \%)$ & 0 & \multirow{3}{*}{$<0.05$} \\
\hline \multirow{2}{*}{$\begin{array}{c}\text { Pre-operative serum } \\
\text { PSA level }\end{array}$} & $4-10 \mathrm{ng} / \mathrm{ml}$ & 41 & $4(9.8 \%)$ & $21(51.2 \%)$ & $12(29.3 \%)$ & $4(9.8 \%)$ & \\
\hline & $>10 \mathrm{ng} / \mathrm{ml}$ & 34 & $6(17.6 \%)$ & $4(11.8 \%)$ & $11(32.4 \%)$ & $\begin{array}{c}13 \\
(38.2 \%)\end{array}$ & \\
\hline \multicolumn{2}{|c|}{ Prostatic carcinoma cases } & 50 & $\begin{array}{c}6 / 50 \\
(12 \%)\end{array}$ & $\begin{array}{l}13 / 50 \\
(26 \%)\end{array}$ & $\begin{array}{l}16 / 50 \\
(32 \%)\end{array}$ & $\begin{array}{l}15 / 50 \\
(30 \%)\end{array}$ & \multirow{7}{*}{$<0.01$} \\
\hline \multirow{9}{*}{$\begin{array}{c}\text { Gleason grade group of } \\
\text { PCa cases }\end{array}$} & Score 6 & 12 & 2 (16.7\%) & $8(66.6 \%)$ & 2 (16.7\%) & 0 & \\
\hline & Score 7 & 22 & 3 (13.6\%) & $4(18.2 \%)$ & 12 (54.6\%) & $3(13.6 \%)$ & \\
\hline & Score 8 & 7 & $1(14.3 \%)$ & $1(14.3 \%)$ & $1(14.3 \%)$ & 4 (57.1\%) & \\
\hline & Score 9 & 9 & 0 & 0 & 1 (11.1\%) & 8 (88.9\%) & \\
\hline & Grade I & 12 & $2(16.7 \%)$ & $8(66.6 \%)$ & $2(16.7 \%)$ & 0 & \\
\hline & Grade II & 14 & $2(14.3 \%)$ & $3(21.4 \%)$ & 8 (57.1\%) & 1 (7.1\%) & \\
\hline & Grade III & 8 & $1(12.5 \%)$ & $1(12.5 \%)$ & $4(50 \%)$ & $2(25 \%)$ & \multirow[t]{3}{*}{$<0.01$} \\
\hline & Grade IV & 7 & $1(14.3 \%)$ & $1(14.3 \%)$ & $1(14.3 \%)$ & 4 (57.1\%) & \\
\hline & Grade V & 9 & 0 & 0 & 1 (11.1\%) & $8(88.9 \%)$ & \\
\hline \multirow{2}{*}{$\begin{array}{c}\text { Capsular invasion in } \\
\text { prostatectomy } \\
\text { specimens only }\end{array}$} & Present & $\begin{array}{c}17 / 2 \\
5\end{array}$ & $\begin{array}{c}3 \\
(17.6 \%)\end{array}$ & $2(11.8 \%)$ & $6(35.3 \%)$ & $\begin{array}{c}6 \\
(35.3 \%)\end{array}$ & \multirow{2}{*}{$>0.05$} \\
\hline & Absent & $8 / 25$ & 0 & $1(12.5 \%)$ & $2(25 \%)$ & $\begin{array}{c}5 \\
(62.5 \%)\end{array}$ & \\
\hline \multirow{2}{*}{$\begin{array}{c}\text { Perineural invasion in } \\
\text { PCa cases }\end{array}$} & Present & 13 & 0 & $2(15.4 \%)$ & $5(38.5 \%)$ & $\begin{array}{c}6 \\
(46.1 \%)\end{array}$ & \multirow[t]{2}{*}{$<0.05$} \\
\hline & Absent & 37 & $6(16.2 \%)$ & $11(29.7 \%)$ & $11(29.7 \%)$ & $9(24.4 \%)$ & \\
\hline \multirow{3}{*}{$\begin{array}{l}\text { Lymphovascular } \\
\text { invasion in PCa cases }\end{array}$} & Present & 20 & $3(15 \%)$ & $2(10 \%)$ & $6(30 \%)$ & $9(45 \%)$ & \multirow{2}{*}{$<0.05$} \\
\hline & Absent & 30 & $3(10 \%)$ & $11(36.7 \%)$ & $10(33.3 \%)$ & $6(20 \%)$ & \\
\hline & $p T 2$ & 29 & $5(17.3 \%)$ & $11(37.9 \%)$ & 11 (37.9\%) & $2(6.9 \%)$ & \multirow[b]{2}{*}{$<0.01$} \\
\hline Pathologic T (pT) & $p T 3$ & 21 & $1(4.8 \%)$ & $2(9.5 \%)$ & $5(23.8 \%)$ & $\begin{array}{c}13 \\
(61.9 \%)\end{array}$ & \\
\hline \multirow{2}{*}{$\begin{array}{c}\text { LN metastasis in } P C a \\
\text { cases }\end{array}$} & Present & 7 & 0 & $1(14.3 \%)$ & $2(28.6 \%)$ & $4(57.1 \%)$ & \\
\hline & Absent & 43 & $6(14 \%)$ & $12(27.9 \%)$ & $14(32.6 \%)$ & $\begin{array}{c}11 \\
(25.5 \%)\end{array}$ & $<0.05$ \\
\hline Distant metastasis in & Present & 4 & 0 & $1(25 \%)$ & $2(50 \%)$ & $1(25 \%)$ & \\
\hline $\begin{array}{l}\text { PCa cases } \\
\text { PCo }\end{array}$ & Absent & 46 & $6(13 \%)$ & $12(26.2 \%)$ & $14(30.4 \%)$ & $\begin{array}{c}14(30.4 \\
\%)\end{array}$ & $>0.05$ \\
\hline & Stage I & 9 & $2(22.2 \%)$ & $5(55.6 \%)$ & $2(22.2 \%)$ & 0 & \\
\hline Tumor stage of PCa & Stage II & 20 & $3(15 \%)$ & $6(30 \%)$ & $9(45 \%)$ & $2(10 \%)$ & $<0,01$ \\
\hline cases & Stage III & 11 & 1 (9.1\%) & 0 & $1(9.1 \%)$ & $9(81.8 \%)$ & $<0.01$ \\
\hline & Stage IV & 10 & 0 & $2(20 \%)$ & $4(40 \%)$ & $4(40 \%)$ & \\
\hline
\end{tabular}




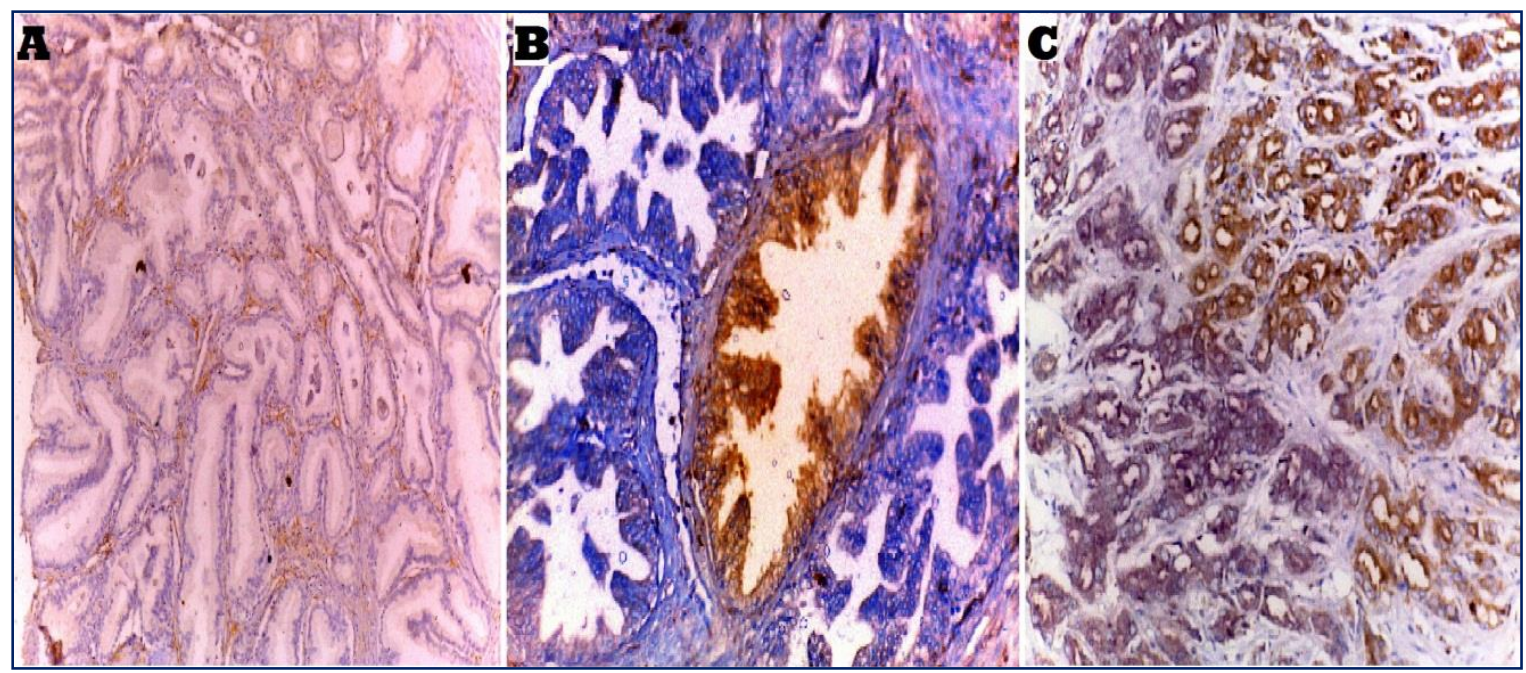

Figure 1: A: Benign prostatic hyperplasia (BPH) showing negative PSCA expression (Avidin-biotin complex x100). B: High grade prostatic intraepithelial neoplasia (HGPIN) showing weak (1+) PSCA cytoplasmic expression (Avidin-biotin complex x200). C: Prostatic carcinoma, Gleason score 7 (Grade group II) showing moderate (2+) PSCA cytoplasmic expression (Avidin-biotin complex x200).

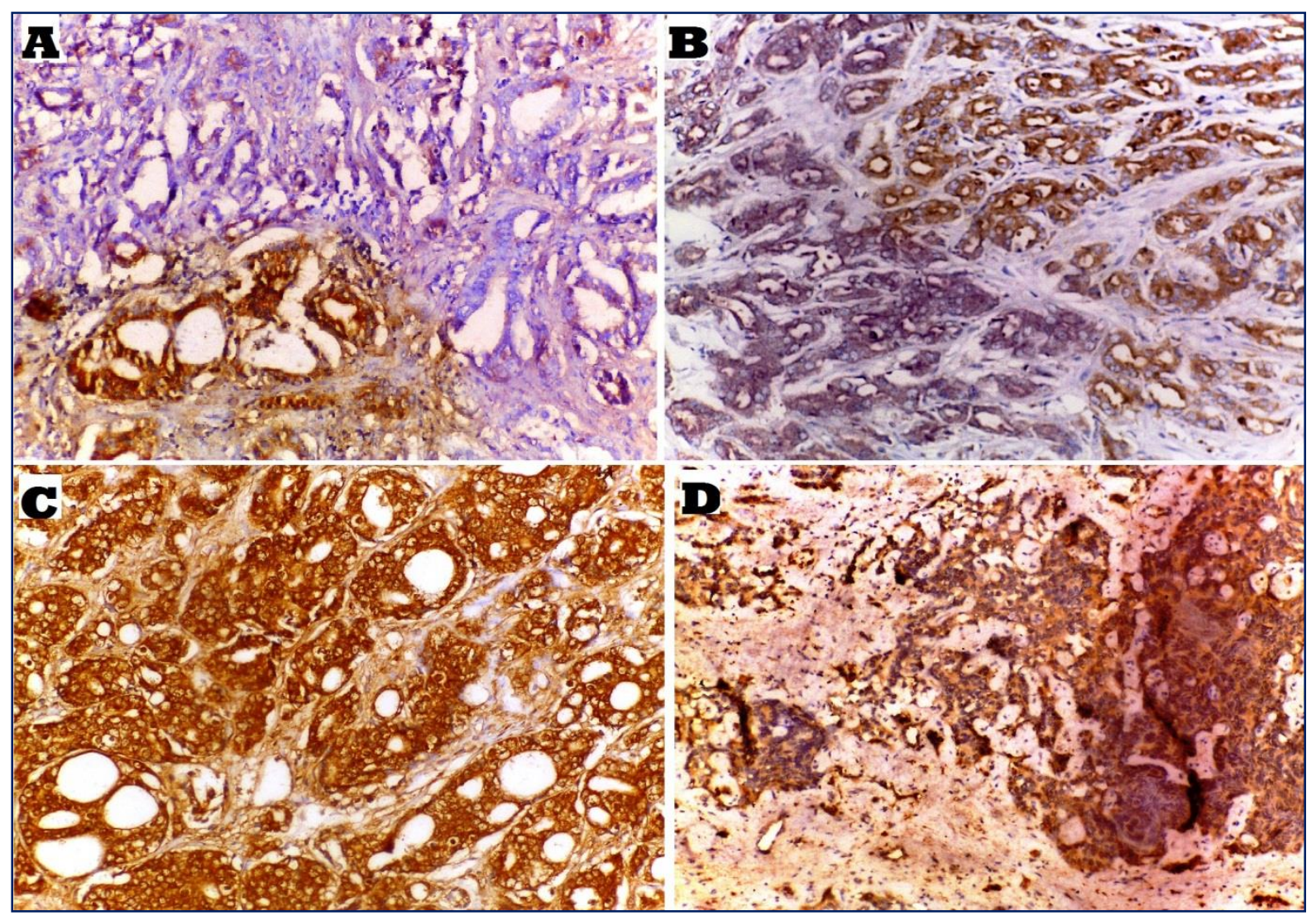

Figure 2: A: Prostatic carcinoma, Gleason score $6(3+3)$ (Grade group I) showing weak (1+) PSCA cytoplasmic expression (Avidin-biotin complex x200). B: Prostatic carcinoma, Gleason score 7 (3+4) (Grade group II) showing moderate (2+) PSCA cytoplasmic expression (Avidin-biotin complex x200). C: Prostatic carcinoma, Gleason score 8 (4+4) (Grade group IV) showing strong (3+) PSCA cytoplasmic expression (Avidin-biotin complex x200). D: Prostatic carcinoma, Gleason score $9(5+4)$ (Grade group V) showing strong (3+) PSCA cytoplasmic expression (Avidin-biotin complex x200). 
Benha medical journal vol.38, academic issue, 2021

Table (3): Relation between the score of Snail expression and clinico-pathological parameters of prostatic carcinoma:

\begin{tabular}{|c|c|c|c|c|c|c|c|}
\hline \multirow{2}{*}{$\begin{array}{c}\text { Clinico-pathological } \\
\text { parameter }\end{array}$} & \multirow{2}{*}{$\begin{array}{l}\text { Categories } \\
\text { of the } \\
\text { parameter }\end{array}$} & \multirow{2}{*}{$\begin{array}{c}\text { No. } \\
o f \\
\text { cases }\end{array}$} & \multicolumn{4}{|c|}{ Score of Snail expression } & \multirow[b]{2}{*}{ P-value } \\
\hline & & & Negative & Score $(1)$ & Score (2) & Score (3) & \\
\hline \multirow{2}{*}{ Studied cases } & & 80 & $19 / 80$ & $25 / 80$ & $19 / 80$ & $17 / 80$ & \multirow{6}{*}{$<0.01$} \\
\hline & & 80 & $(23.75 \%)$ & (31.25\%) & $(23.75 \%)$ & $(21.25 \%)$ & \\
\hline \multirow{4}{*}{$\begin{array}{l}\text { Histopathological type of } \\
\text { the prostatic lesion }\end{array}$} & BPH & 17 & $7(41.2 \%)$ & $9(52.9 \%)$ & $1(5.9 \%)$ & 0 & \\
\hline & HGPIN & 13 & $4(30.8 \%)$ & $5(38.4 \%)$ & $3(23.1 \%)$ & $1(7.7 \%)$ & \\
\hline & $P C a$ & 50 & $8(16 \%)$ & $11(22 \%)$ & $15(30 \%)$ & $16(32 \%)$ & \\
\hline & $<40$ years & 8 & $3(37.5 \%)$ & 0 & $3(37.5 \%)$ & $2(25 \%)$ & \\
\hline \multirow{2}{*}{ Age } & 40-65 years & 34 & $2(5.9 \%)$ & $17(50 \%)$ & $8(23.5 \%)$ & $7(20.6 \%)$ & \multirow{2}{*}{$>0.05$} \\
\hline & $>65$ years & 38 & $\begin{array}{c}14 \\
(36.7 \%)\end{array}$ & $8(21.1 \%)$ & $8(21.1 \%)$ & $8(21.1 \%)$ & \\
\hline \multirow{3}{*}{$\begin{array}{c}\text { Pre-operative serum PSA } \\
\text { level }\end{array}$} & $<4 n g / m l$ & 5 & 0 & $1(20 \%)$ & $3(60 \%)$ & $1(20 \%)$ & \multirow{3}{*}{$>0.05$} \\
\hline & $4-10 \mathrm{ng} / \mathrm{ml}$ & 41 & $7(17.1 \%)$ & $18(43.9 \%)$ & $\begin{array}{c}10 \\
(24.4 \%)\end{array}$ & $6(14.6 \%)$ & \\
\hline & $>10 \mathrm{ng} / \mathrm{ml}$ & 34 & $\begin{array}{c}12 \\
(35.4 \%)\end{array}$ & $6(17.6 \%)$ & $6(17.6 \%)$ & $10(29.4 \%)$ & \\
\hline \multicolumn{2}{|c|}{ Prostatic carcinoma cases } & 50 & $\begin{array}{l}8 / 50 \\
(16 \%)\end{array}$ & $\begin{array}{l}11 / 50 \\
(22 \%)\end{array}$ & $\begin{array}{l}15 / 50 \\
(30 \%)\end{array}$ & $\begin{array}{l}16 / 50 \\
(32 \%)\end{array}$ & \multirow{6}{*}{$<0.01$} \\
\hline \multirow{5}{*}{ Gleason score of PCa cases } & Score 6 & 12 & $4(33.3 \%)$ & $5(41.7 \%)$ & $2(16.7 \%)$ & $1(8.3 \%)$ & \\
\hline & Score 7 & 22 & $4(18.2 \%)$ & $5(22.7 \%)$ & 9 (40.9\%) & $4(18.2 \%)$ & \\
\hline & Score 8 & 7 & 0 & $1(14.3 \%)$ & $2(28.6 \%)$ & $4(57.1 \%)$ & \\
\hline & Score 9 & 9 & 0 & 0 & $2(22.2 \%)$ & $7(77.8 \%)$ & \\
\hline & Grade I & 12 & $4(33.3 \%)$ & $5(41.7 \%)$ & $2(16.7 \%)$ & $1(8.3 \%)$ & \\
\hline \multirow{4}{*}{$\begin{array}{c}\text { Gleason grade group of } \mathrm{PCa} \\
\text { cases }\end{array}$} & Grade II & 14 & $3(21.4 \%)$ & $3(21.4 \%)$ & $5(35.8 \%)$ & $3(21.4 \%)$ & \multirow{4}{*}{$<0.01$} \\
\hline & Grade III & 8 & $1(12.5 \%)$ & $2(25 \%)$ & $4(50 \%)$ & $1(12.5 \%)$ & \\
\hline & Grade IV & 7 & 0 & $1(14.3 \%)$ & $2(28.6 \%)$ & $4(57.1 \%)$ & \\
\hline & Grade V & 9 & 0 & 0 & $2(22.2 \%)$ & $7(77.8 \%)$ & \\
\hline $\begin{array}{l}\text { Capsular invasion in } \\
\text { prostatectomy specimens }\end{array}$ & Present & $\begin{array}{c}17 / 2 \\
5\end{array}$ & $3(17.6 \%)$ & $3(17.6 \%)$ & $5(29.4 \%)$ & $6(35.3 \%)$ & \multirow[t]{2}{*}{$>0.05$} \\
\hline only & Absent & $8 / 25$ & $2(25 \%)$ & $1(12.5 \%)$ & $2(25 \%)$ & $3(37.5 \%)$ & \\
\hline \multirow{2}{*}{$\begin{array}{c}\text { Perineural invasion in PCa } \\
\text { cases }\end{array}$} & Present & 13 & $1(7.7 \%)$ & $1(7.7 \%)$ & $\begin{array}{c}5 \\
(38.5 \%)\end{array}$ & $6(46.1 \%)$ & \multirow{2}{*}{$>0.05$} \\
\hline & Absent & 37 & $7(19 \%)$ & $10(27 \%)$ & $10(27 \%)$ & $10(27 \%)$ & \\
\hline \multirow{2}{*}{$\begin{array}{c}\text { Lymphovascular invasion in } \\
\text { PCa cases }\end{array}$} & Present & 20 & $4(20 \%)$ & $2(10 \%)$ & $6(30 \%)$ & $8(40 \%)$ & \multirow{2}{*}{$>0.05$} \\
\hline & Absent & 30 & $4(13.3 \%)$ & $9(30 \%)$ & $9(30 \%)$ & $8(26.7 \%)$ & \\
\hline \multirow{2}{*}{ Pathologic $T(p T)$} & $p T 2$ & 29 & $6(20.7 \%)$ & $9(31 \%)$ & $\begin{array}{c}8 \\
(27.6 \%)\end{array}$ & $6(20.7 \%)$ & \multirow{2}{*}{$<0.05$} \\
\hline & $p T 3$ & 21 & $2(9.5 \%)$ & $2(9.5 \%)$ & $\begin{array}{c}7 \\
(33.3 \%)\end{array}$ & $10(47.7 \%)$ & \\
\hline \multirow[b]{2}{*}{ LN metastasis in PCa cases } & Present & 7 & 0 & 0 & $2(28.6 \%)$ & 5 (71.4\%) & \multirow[b]{2}{*}{$<0.05$} \\
\hline & Absent & 43 & $8(14 \%)$ & $\begin{array}{c}11 \\
(25.5 \%)\end{array}$ & $\begin{array}{c}13 \\
(30.2 \%)\end{array}$ & $11(25.4 \%)$ & \\
\hline Distant metastasis in PCa & Present & 4 & 0 & $1(25 \%)$ & $1(25 \%)$ & $2(50 \%)$ & \\
\hline $\begin{array}{c}\text { Distant metastasis in PCa } \\
\text { cases }\end{array}$ & Absent & 46 & $8(17.4 \%)$ & $10(21.8 \%)$ & $\begin{array}{c}14 \\
(30.4 \%)\end{array}$ & $14(30.4 \%)$ & $>0.05$ \\
\hline & Stage I & 9 & $1(11.1 \%)$ & $3(33.3 \%)$ & $4(44.4 \%)$ & $1(11.1 \%)$ & \\
\hline Tumor stage of PCa casos & Stage II & 20 & $5(25 \%)$ & $6(30 \%)$ & $4(20 \%)$ & $5(25 \%)$ & $<005$ \\
\hline Tumor stage of PCa cases & Stage III & 11 & $2(18.1 \%)$ & $1(9.1 \%)$ & $4(36.4 \%)$ & $4(36.4 \%)$ & $<0.05$ \\
\hline & Stage IV & 10 & 0 & $1(10 \%)$ & $3(30 \%)$ & $6(60 \%)$ & \\
\hline
\end{tabular}




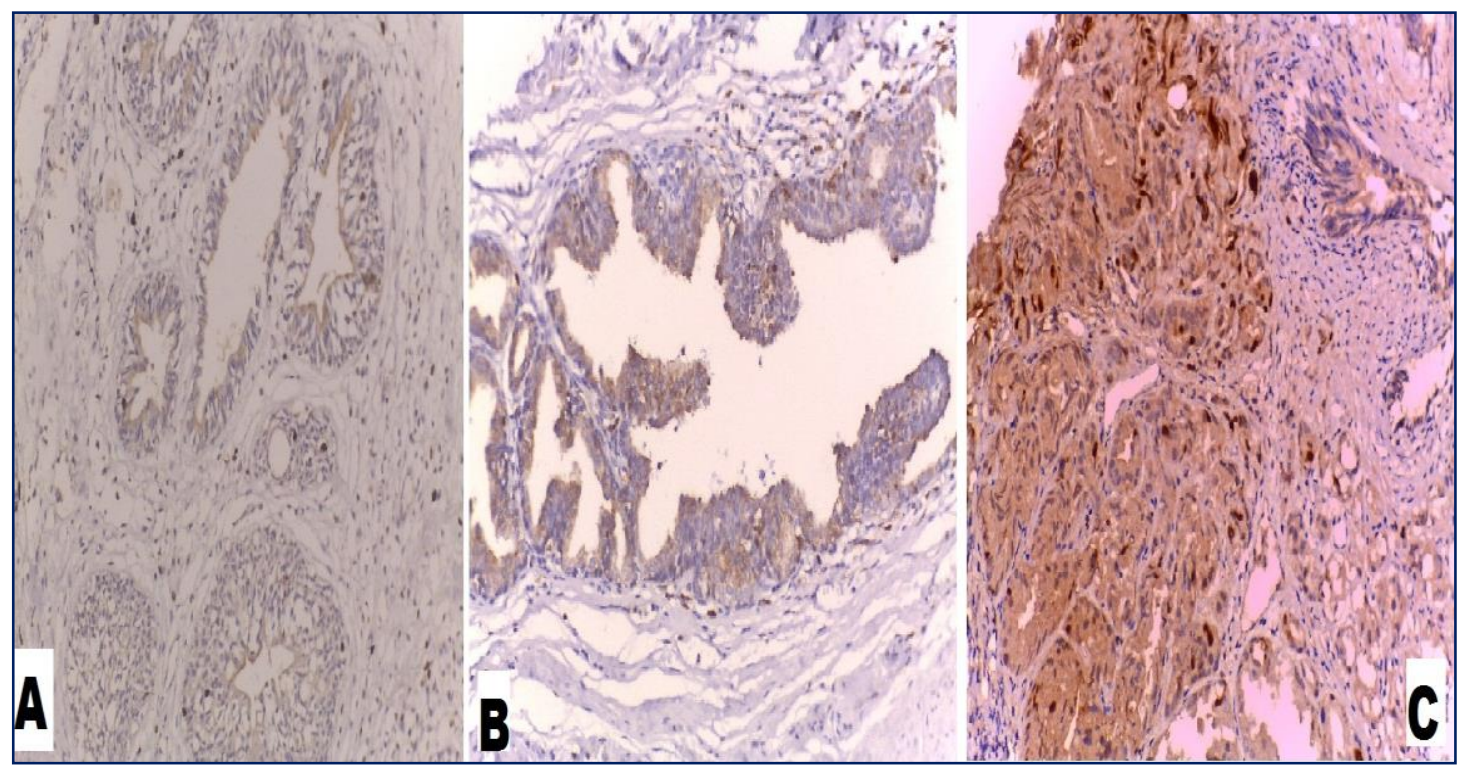

Figure 3: A: Benign prostatic hyperplasia (BPH) showing negative nuclear Snail expression (Avidin-biotin complex x200). B: High grade prostatic intraepithelial neoplasia (HGPIN) showing weak (score 1) Snail nuclear expression (Avidin-biotin complex x200). C: Prostatic carcinoma, Gleason score 7 (3+4) (Grade group II) showing moderate (score 2) Snail nuclear expression (Avidin-biotin complex x200).

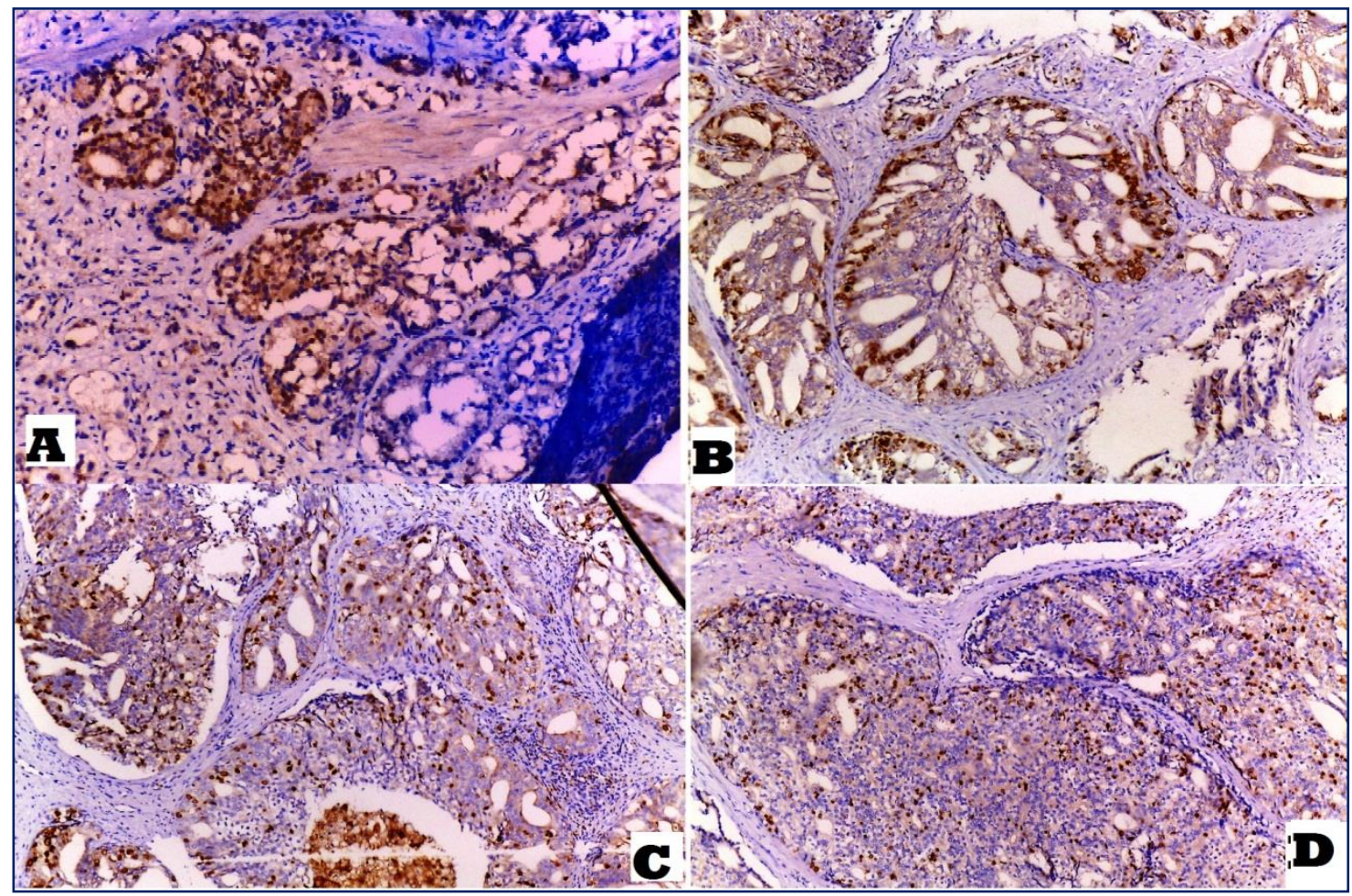

Figure 4: A: Prostatic carcinoma, Gleason score $7(4+3)$ (Grade group II) showing moderate (score 2) Snail nuclear expression (Avidin-biotin complex x100). B: Prostatic carcinoma, Gleason score 8 (4+4) (Grade group IV) showing strong (score 3) Snail nuclear expression (Avidin-biotin complex x100). C: Prostatic carcinoma, Gleason score $9(4+5)$ (Grade group V) showing strong (score 3) Snail nuclear expression (Avidin-biotin complex x100). D: Prostatic carcinoma, Gleason score $10(5+5)$ (Grade group V) showing strong (score 3) Snail nuclear expression (Avidin-biotin complex x100). 


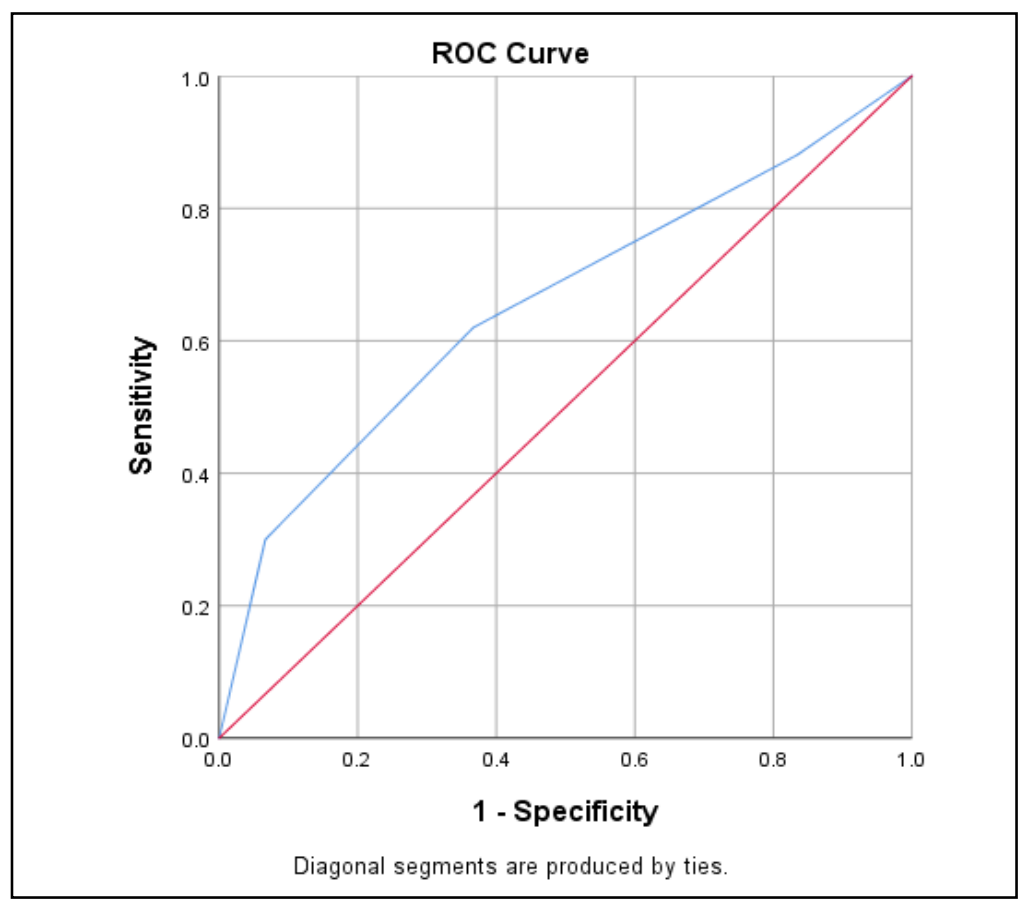

Figure (5): Receiver-operating characteristic (ROC) to predict sensitivity, specificity and accuracy of PSCA immunohistochemical score

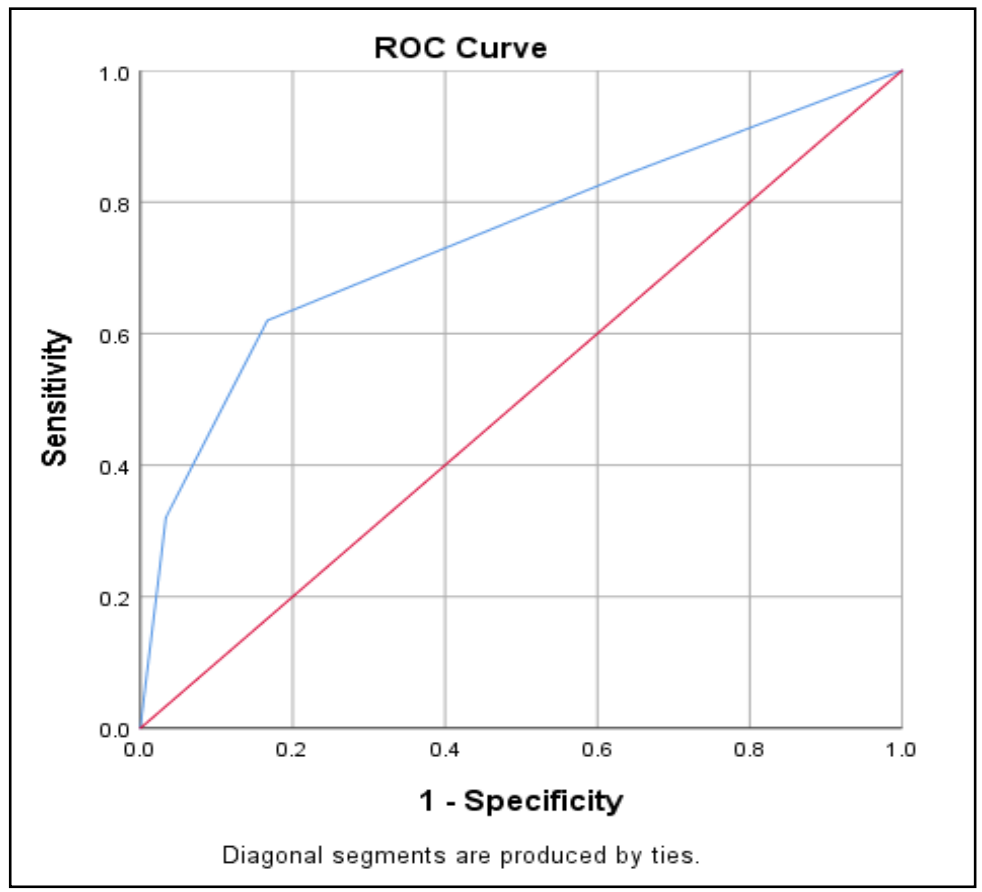

Figure (6): Receiver-operating characteristic (ROC) to predict sensitivity, specificity and accuracy of Snail immunohistochemical score 
Table (4): Validity of immunohistochemical score of both PSCA and Snail in differentiating between different prostatic lesions:

\begin{tabular}{lcc}
\hline & PSCA & Snail \\
\hline Sensitivity & $58.0 \%$ & 62.0 \\
Specificity & $63.3 \%$ & 83.3 \\
Positive Predictive Value (PPV) & 72.5 & 86.1 \\
Negative Predictive Value (NPV) & 47.5 & 56.8 \\
Accuracy & 60.0 & 70.0 \\
Statistical test (x2) & 3.41 & 15.57 \\
P value & 0.065 & $<0.001^{* *}$ \\
\hline
\end{tabular}

Table (5): Relation between the score of PSCA expression and Snail expression in the studied cases:

\begin{tabular}{lcccccc}
\hline $\begin{array}{c}\text { Score of PSCA } \\
\text { Expression }\end{array}$ & $\begin{array}{c}\text { Negative } \\
(\mathbf{0})\end{array}$ & $\begin{array}{c}\text { Weak } \\
\text { Expression } \\
(\mathbf{1 + )}\end{array}$ & $\begin{array}{c}\text { Moderate } \\
\text { Expression } \\
(\mathbf{2 +})\end{array}$ & $\begin{array}{c}\text { Strong } \\
\text { Expression } \\
(\mathbf{3 +})\end{array}$ & Total & P-value \\
$\begin{array}{c}\text { Score of Snail } \\
\text { expression }\end{array}$ & & & & & & \\
\hline Negative (Score 0) & $6(31.6 \%)$ & $8(42.1 \%)$ & $2(10.5 \%)$ & $3(15.8 \%)$ & $\mathbf{1 9}$ & \\
Score 1 & $1(4 \%)$ & $13(52 \%)$ & $10(40 \%)$ & $1(4 \%)$ & $\mathbf{2 5}$ & \\
Score 2 & $2(10.5 \%)$ & $4(21.05 \%)$ & $9(47.3 \%)$ & $4(21.05 \%)$ & $\mathbf{1 9}$ & $<0.01$ \\
Score 3 & $2(11.8 \%)$ & $2(11.8 \%)$ & $4(23.5 \%)$ & $9(52.9 \%)$ & $\mathbf{1 7}$ & \\
Total & $\mathbf{1 1 ( 1 3 . 7 5 \% )}$ & $\mathbf{2 7 ( 3 3 . 7 5 \% )}$ & $\mathbf{2 5 ( 3 1 . 2 5 \% )}$ & $\mathbf{1 7 ( 2 1 . 2 5 \% )}$ & $\mathbf{8 0}$ & \\
\hline
\end{tabular}

\section{Discussion:}

Prostatic carcinoma is a common malignancy, representing the 2nd leading cause of cancer death in America, and the 5th cause worldwide (22). Its incidence is rising rapidly with popularization of the PSA-based screening for PCa (10).

In Egypt it was reported that $\mathrm{PCa}$ represents $4.27 \%$ of total cancers among men and $60.7 \%$ of male genital caners (23)
This current retrospective study was done on 80 cases of different prostatic lesions; BPH, HGPIN and PCa. Each case was immunohistochemically stained and evaluated for PSCA and Snail expression. The expression of both markers was assessed in relation to different histopathological variables of $\mathrm{PCa}$ and with each other.

The mean age of BPH, HGPIN, and PCa cases was 60, 65, 65.5 years respectively. 
This agreed with a study which reported that PCa was seen in older age than benign lesions, and there was an increased incidence of malignancy with advancing age (24).

In this current study, the mean value of PSA in BPH, HGPIN and PCa cases was $(7.3 \mathrm{ng} / \mathrm{ml}, \quad 13.1 \mathrm{ng} / \mathrm{ml}$, and $23.5 \mathrm{ng} / \mathrm{ml}$ respectively) with increasing level from benign to malignant lesions. This agreed with the study which found that BPH and PIN cases had PSA ranging 0-7ng/ml, while PCa cases had PSA > 20ng/ml. This concluded that an increasing PSA level could imply underlying malignancy (25).

The Gleason grade of studied PCa cases showed a highly significant statistical correlation with pathologic $\mathrm{T}$ (Pvalue<0.01), and a significant statistical correlation with age, PSA, peri-neural and lymphovascular invasion ( $\mathrm{P}$-value $<0.05)$. It was reported that PCa patients aged $>75$ years had higher PSA levels and were more liable to have high grade tumors with extra-prostatic extension (26) and another study reported that lymphovascular invasion usually presents in high grade $\mathrm{PCa}$ (27).

In this study, PCa grade showed a highly significant statistical correlation with the stage (P-value<0.01). Also, it was found that larger tumors in radical prostatectomy tend to have higher grade, and stage (28).

Prostatic Stem Cell Antigen (PSCA) is a small, GPI-anchored cell surface protein belonging to the Thy-1/Ly-6 family. It was recognized in several primary cancers including bladder, pancreatic, gastric, and non-small-cell lung carcinoma (10).

In this study, PSCA expression showed a highly significant statistical correlation with histopathological type of the lesion (P-value $<0.01)$. This agreed with many studies (19 \& 29) where it was found that PSCA expression was stronger in malignant prostatic cells than adjacent benign tissues. Thus PSCA seemed to have a role in prostatic tumorigenesis.

In this study, PSCA expression in $\mathrm{PCa}$ cases showed a highly significant statistical correlation with Gleason score, pathologic $\mathrm{T}$, tumor grade, and stage ( $\mathrm{P}$ value $<0.01$ ), and a significant statistical correlation with PSA, lymph node metastasis, peri-neural and lymphovascular invasion ( $\mathrm{P}$-value $<0.05)$.

Those results agreed with others $(\mathbf{1 9} \mathbf{8 3 0})$ where it was reported that PSCA overexpression was positively correlated with advanced clinical stage, seminal 
vesicle and capsular invasion. In addition, it was found that PSCA knockdown in bladder carcinoma was associated with reduced cancer cell proliferation in vitro and in vivo (31).

The effect of PSCA on migratory and invasiveness abilities of PCa cells was examined and it was found that migration of malignant cells was significantly promoted by PSCA overexpression, and decreased by PSCA knockdown. Thus, PSCA is suggested to promote migration and invasion of PCa cells (32).

The proto-oncogene c-Myc had an impact on cell proliferation and differentiation, and its amplification played a role in early prostate epithelial cell transformation (33). A correlation was found between PSCA and c-Myc protein levels in $\mathrm{PCa}$ tissues, and that PSCA promotes cell cycle progression via up-regulating c-Myc expression. PI3K/AKT signaling pathways were found in their study to be involved in PSCA-mediated c-Myc expression and PCa growth (19).

In contrast, it was demonstrated that SOX5 is an important regulatory repressor of PSCA gene in esophageal squamous cell carcinoma cells and PSCA overexpression induced cell cycle arrest and promoted cell differentiation (34). Also, the cell growth-inhibitory activity of PSCA in gallbladder carcinoma was and it seemed that biological function of PSCA in tumor growth is tissue and cell-type dependent (35)..

Snail is a transcription factor belonging to the zinc finger family proteins (36). In this study, Snail expression showed a highly significant statistical correlation with histopathological type of the lesion (Pvalue $<0.01)$. This agreed with the study done on 2015 that found that positive Snail nuclear immunostaining was detected in $53.8 \%$ of $\mathrm{PCa}$ specimens versus none of BPH cases $(\mathrm{P}<0.001)$. Moreover, HGPIN foci showed weak Snail expression, while benign prostatic tissues were completely negative irrespective of the level of Snail expression within the malignant tissue (37).

It was found that snail expression is higher in gastric cancer tissues than in para-carcinoma and normal tissues(38). Moreover, Snail was reported to be highly expressed in several carcinomas including ovarian, urothelial, breast, hepatocellular, gastric, and non-small cell lung carcinomas (39). Thus Snail may have a role in tumorigenesis. 
In this study, Snail expression in $\mathrm{PCa}$ cases showed a highly significant statistical correlation with Gleason score and tumor grade (P-value<0.01).This agreed with the study which proved that high Gleason grades show higher Snail expression than low Gleason grade samples (40). Also, it was noticed that patients with increased Snail expression had higher Gleason scores and tumor volume than those with low expression (41).

In contrast, it was reported that Snail was expressed in high levels without significant differences between colorectal carcinomas, adenomas and histologically normal adjacent mucosa (42).

In this study, Snail expression showed a significant statistical correlation with pathologic T, lymph node metastasis, and tumor stage $(\mathrm{P}$-value $<0.05)$. This agreed with other studies that found that Snail immunostaining was significantly higher in PCa with lymph node metastasis than those without nodal metastasis, and an association was detected between positive Snail immunostaining and higher TNM stages (37).

In addition, it was observed that Snail expression was higher in gastric carcinoma with lymphatic metastasis, lower differentiation, and late clinical stage. This concluded that Snail is significantly associated with tumor progression and metastasis in gastric carcinoma (38).

In a study carried out on 2018, Snail was significantly higher in the late stage of primary ovarian cancer and metastatic lesions than in early-stage tumors and that Snail expression and localization was inversely correlated with E-cadherin (cellcell adhesion molecule) (43).

It was noticed that high levels of Snail closely correlated with lymph node and distant metastasis in pancreatic adenocarcinoma, and Snail knockdown resulted in the reversal of epithelialmesenchymal transition (EMT) in carcinoma cells (44).

Many studies found that Snail has a major role in tumor invasion, metastasis and progression through induction of epithelial-mesenchymal transition by inhibiting the expression of epithelial markers like E-cadherin by binding to the E-box region within the E-cadherin promoter and represses its transcription, and simultaneously promotes 
mesenchymal markers expression like Vimentin and N-cadherin (45).

The ectopic expression of Snail enhanced the expression of VEGFA, and endothelial markers like CD31 and VEGFR2. Therefore, Snail enhanced tumor progression not only through its tumorinitiating capacity, but also through its ability to promote angiogenesis, suggesting that it may be a promising target for cancer therapy (45)

In this study, receiver-operating characteristic (ROC) curve showed that Snail is more valid than PSCA in differentiating between cancerous and non-cancerous prostatic lesions; as the PPV was 86.1 and 72.5 respectively.

In this study, there was a highly significant statistical correlation between the score of PSCA and Snail expression in the studied lesions (P-value<0.01). Thus, PSCA and Snail may be used as a predictive co-biomarker for patient prognosis and tumor aggressiveness in PCa.

To our knowledge, this is the first study demonstrating a significant correlation between PSCA and Snail regarding their immunohistochemical expression in different prostatic lesions.

\section{Conclusion}

The present work reveals that expression of PSCA and Snail increased from BPH to HGPIN to PCa so they may have a role in prostatic tumorigenesis. Also, their expression increased with high grade, advanced stage, and metastatic prostatic carcinoma. Thus, they could be considered potentially prognostic markers for further confirmation by larger survival analysis.

\section{References}

1. Vuichoud $\mathrm{C}$ and Loughlin KR. Benign prostatic hyperplasia: epidemiology, economics and evaluation. Can J Urol. 2015 Oct;22(Suppl 1):1-6.

2. Friedman P, Costa D, Kapur P. Foamy gland high-grade prostatic intraepithelial neoplasia on core biopsy and subsequent radical prostatectomy: An in depth case report of a rare variant. Human Pathology: Case Reports. 2017 Nov 1;10:32-6.

3. Brimo F. High-Grade Prostatic Intraepithelial Neoplasia. InPrecision Molecular Pathology of Prostate Cancer 2018 (pp. 27-36).

4. Bray F, Ferlay J, Soerjomataram I, Siegel RL, Torre LA, Jemal A. Global cancer statistics 2018: GLOBOCAN estimates of incidence and mortality worldwide for 36 cancers in 185 countries. CA: a cancer journal for clinicians. 2018 Nov;68(6):394424.

5. Gray PJ, Lin CC, Cooperberg MR, Jemal A, Efstathiou JA. Temporal trends and the impact of race, insurance, and socioeconomic status in the management of localized 
prostate cancer. European Urology. 2017 May 1;71(5):729-37.

6. Cooperberg MR. and Chan JM. "Epidemiology of prostate cancer." (2017): 849-849.

7. El-Bolkainy MN. "Golden rules in practice of cancer pathology." (2016): 137-140.

8. Kgatle MM, Kalla AA, Islam MM, Sathekge M, Moorad R. Prostate cancer: epigenetic alterations, risk factors, and therapy. Prostate Cancer. 2016 Oct;2016.

9. Lee AR, Li Y, Xie N, Gleave ME, Cox ME, Collins CC, et al. Alternative RNA splicing of the MEAF6 gene facilitates neuroendocrine prostate cancer progression. Oncotarget. 2017 Apr 25;8(17):27966.

10. Xiang Q, Zhu Z, Luo L, Wang J, Liu Y, Deng $\mathrm{Y}$, et al. The Correlation between PSCA Expression and Neuroendocrine Differentiation in Prostate Cancer. BioMed Research International. 2020 Sep 24;2020.

11. Youssef NS, Radwan NA, Abd El Khalek SM, Shahin MA, El-Maraghy MN. Study of immunohistochemical expression of prostate stem cell antigen in prostatic carcinoma. Egyptian Journal of Pathology. 2015 Jul 1;35(1):30-7.

12. Montanari M, Rossetti S, Cavaliere C, D'Aniello C, Malzone MG, Vanacore D, et al. Epithelial-mesenchymal transition in prostate cancer: an overview. Oncotarget. 2017 May 23;8(21):35376.

13. Xu R, Won JY, Kim CH, Kim DE, Yim H. Roles of the phosphorylation of transcriptional factors in epithelialmesenchymal transition. Journal of oncology. 2019 Oct;2019.

14. Edwards G, Campbell T, Henderson V, Danaher A, Wu D, Srinivasan R, et al. SNAIL Transctiption factor in prostate cancer cells promotes neurite outgrowth. Biochimie. 2020 Oct 24;180:1-9.
15. Kang R, Zhao S, Liu L, Li F, Li E, Luo L, et al. Knockdown of PSCA induces EMT and decreases metastatic potentials of the human prostate cancer DU145 cells. Cancer Cell Int. 2016 Mar 15;16:20. doi: 10.1186/s12935016-0295-4. PMID: 26981049; PMCID: PMC4791869.

16. Humphrey PA, Moch H, Cubilla AL, Ulbright TM, Reuter VE. The 2016 WHO classification of tumours of the urinary system and male genital organs-part B: prostate and bladder tumours. European urology. $2016 \mathrm{Jul}$ 1;70(1):106-19.

17. van Leenders GJ, van der Kwast $\mathrm{TH}$, Grignon DJ, Evans AJ, Kristiansen G, Kweldam CF, et al. The 2019 International Society of Urological Pathology (ISUP) Consensus Conference on Grading of Prostatic Carcinoma. The American Journal of Surgical Pathology. 2020 May 26.

18. Buyyounouski MK, Choyke PL, McKenney JK, Sartor O, Sandler HM, Amin MB, et al. Prostate cancer-major changes in the American Joint Committee on Cancer eighth edition cancer staging manual. CA: a cancer journal for clinicians. 2017 May 6;67(3):24553.

19. Li E, Liu L, Li F, Luo L, Zhao S, Wang J, et al. PSCA promotes prostate cancer proliferation and cell-cycle progression by up-regulating c-Myc. The Prostate. 2017 Dec;77(16):1563-72.

20. Papanikolaou S, Bravou V, Papadaki H, Gyftopoulos K. The role of the endothelin axis in promoting epithelial to mesenchymal transition and lymph node metastasis in prostate adenocarcinoma. Urol Ann. 2017 Oct-Dec;9(4):372-379. doi: 10.4103/UA.UA_43_17. PMID: 29118542; PMCID: PMC5656965.

21. Ruan Y, Yu W, Cheng F, Zhang X, Larré S. Detection of prostate stem cell antigen expression in human prostate cancer using quantum-dot-based technology. Sensors. 2012 May;12(5):5461-70. 
22. Siegel RL, Miller KD, Jemal A. Cancer statistics, 2019. CA: a cancer journal for clinicians. 2019 Jan;69(1):7-34.

23. Ibrahim NH, Abdellateif MS, Kassem SH, Abd El Salam MA, El Gammal MM. Diagnostic significance of miR-21, miR-141, miR-18a and miR-221 as novel biomarkers in prostate cancer among Egyptian patients. Andrologia. 2019 Nov;51(10):e13384.

24. Hirachand S, Dangol UM, Pradhanang S, Acharya S. Study of prostatic pathology and its correlation with prostate specific antigen level. Journal of Pathology of Nepal. 2017 Mar 30;7(1):1074-7.

25. Banerji JS, Wolff EM, Massman III JD, Odem-Davis K, Porter CR, Corman JM. Prostate needle biopsy outcomes in the era of the US Preventive Services Task Force recommendation against prostate specific antigen based screening. The Journal of urology. 2016 Jan 1;195(1):66-73.

26. Herlemann A, Buchner A, Kretschmer A, Apfelbeck M, Stief CG, Gratzke C, et al. Postoperative upgrading of prostate cancer in men $\geq 75$ years: a propensity score-matched analysis. World Journal of Urology. 2017 Oct 1;35(10):1517-24.

27. Jiang $\mathrm{W}$, Zhang L, Wu B, Zha Z, Zhao H, Jun Y, et al. The impact of lymphovascular invasion in patients with prostate cancer following radical prostatectomy and its association with their clinicopathological features: An updated PRISMA-compliant systematic review and meta-analysis. Medicine. 2018 Dec;97(49).

28. Schoots IG, Osses DF, Drost FJ, Verbeek JF, Remmers S, van Leenders GJ, et al. Reduction of MRI-targeted biopsies in men with low-risk prostate cancer on active surveillance by stratifying to PI-RADS and PSA-density, with different thresholds for significant disease. Translational andrology and urology. 2018 Feb;7(1):132.

29. Taeb J, Asgari M, Abolhasani M, Farajollahi MM, Madjd Z. Expression of prostate stem cell antigen (PSCA) in prostate cancer: a tissue microarray study of Iranian patients. Pathology-Research and Practice. 2014 Jan 1;210(1):18-23.

30. Kawaguchi T, Sho M, Tojo T, Yamato I, Nomi T, Hotta K, et al. Clinical significance of prostate stem cell antigen expression in non-small cell lung cancer. Japanese journal of clinical oncology. 2015;40(4):319-26.

31. Marra E, Uva P, Viti V, et al. Growth delay of human bladder cancer cells by Prostate Stem Cell Antigen downregulation is associated with activation of immune signaling pathways. Bmc Cancer. 2015; 10:129.

32. Liu L, Li E, Luo L, Zhao S, Li F, Wang J, et al. PSCA regulates IL-6 expression through p38/NF- $\mathrm{kB}$ signaling in prostate cancer. The Prostate. 2017 Oct; 77(14):1389-400.

33. Zhang C, Gao C, Xu Y, Zhang Z. CtBP2 could promote prostate cancer cell proliferation through c-Myc signaling. Gene. 2015 Jan 10;546(1):73-9.

34. Zhang LY, Wu JL, Qiu HB, Dong SS, Zhu $\mathrm{YH}$, Lee $\mathrm{VH}$, et al. PSCA acts as a tumor suppressor by facilitating the nuclear translocation of RB1CC1 in esophageal squamous cell carcinoma. Carcinogenesis. 2016 Mar 1;37(3):320-32.

35. Ono H, Hiraoka N, Lee YS, et al. Prostate stem cell antigen, a presumable organdependent tumor suppressor gene, is downregulated in gallbladder carcinogenesis. Genes Chromosomes Cancer. 2017; 51:30-41.

36. Faget J, Groeneveld S, Boivin G, Sankar M, Zangger N, Garcia M, et al. Neutrophils and snail orchestrate the establishment of a protumor microenvironment in lung cancer. Cell reports. 2017 Dec 12;21(11):3190-204.

37. Fawzy AI, Gayyed MF, Elsaghir GA, Elbadry MS. Expression of Snail transcription factor in prostatic adenocarcinoma in Egypt: correlation with Maspin protein expression and clinicopathologic variables. International Journal of Clinical and Experimental Pathology. 2015,(8):1558-1566. SSN:19362625/IJCEP1305031. 
38. Chen X, Li J, Hu L, Yang W, Lu L, Jin H, et al. The clinical significance of snail protein expression in gastric cancer: a meta-analysis. Human genomics. $2016 \mathrm{Jul}$ 1;10(2):22.

39. Wang G, Ma W, Li Y, Jiang Y, Ma G, Zhang $X$, et al. Prognostic value of Twist, Snail and E-cadherin expression in pathological NO non-small-cell lung cancer: a retrospective cohort study. European Journal of CardioThoracic Surgery. 2018 Aug 1;54(2):237-45.

40. Edwards G, Campbell T, Henderson V, Danaher A, Wu D, Srinivasan R, et al. SNAIL Transctiption factor in prostate cancer cells promotes neurite outgrowth. Biochimie. 2020 Oct 24;180:1-9.

41. Ipekci T, Ozden F, Unal B, Saygin C, Uzunaslan D and Ates E. EpithelialMesenchymal transition markers $\beta$-catenin, snail, and E-Cadherin do not predict disease free survival in prostate adenocarcinoma: a prospective study. Pathology \& Oncology Research. 2015 Sep 1;21(4):1209-16.

42. Bezdekova M, Brychtova S, Sedlakova E, Langova K, Brychta T, Belej K. Analysis of snail-1, e-cadherin and claudin-1 expression in colorectal adenomas and carcinomas. International journal of molecular sciences. 2015;13(2):1632-43.

43. Ghoneum A, Afify H, Salih Z, Kelly M, Said $\mathrm{N}$. Role of tumor microenvironment in ovarian cancer pathobiology. Oncotarget. 2018 Apr 27;9(32):22832-22849. doi: 10.18632/oncotarget.25126. PMID: 29854318 ; PMCID: PMC5978268.

44. Liu M, Hancock SE, Sultani G, Wilkins BP, Ding E, Osborne B, et al. SnailOverexpression Induces Epithelialmesenchymal Transition and Metabolic Reprogramming in Human Pancreatic Ductal Adenocarcinoma and Non-tumorigenic Ductal Cells. J Clin Med. 2019 Jun 8;8(6):822. doi: 10.3390/jcm8060822. PMID: 31181802; PMCID: PMC6617272.

45. Chang Z, Zhang Y, Liu J, Zheng Y, Li H, Kong Y, et al. Snail promotes the generation of vascular endothelium by breast cancer cells. Cell Death \& Disease. 2020 Jun 15;11(6):1-7.

To cite this article: Marwa S. Abd Allah, Nancy Abo Elgheit Dawood, Ranih Z. Amer, Taghreed Abd Elsamea, Abd Ellatif M. Elbalshy. Role of Prostatic Stem Cell Antigen (PSCA) and Snail in Different Prostatic Lesions (An immunohistochemical Study). BMFJ 2021; 38 (academic issue):166-185. DOI: 10.21608/bmfj.2021.53397.1358 